\title{
Carnets
}

Revue électronique d'études françaises de l'APEF

Deuxième série - $12 \mid 2018$

Théorie Mimétique et Études Littéraires

\section{Sa majesté des Mouches et les bienheureux de la désolation}

Deux cas de mimétisme insulaire aux antipodes?

\section{Éric Fougère}

\section{CpenEdition}

\section{Journals}

Édition électronique

URL : http://journals.openedition.org/carnets/2465

DOI : $10.4000 /$ carnets. 2465

ISSN : 1646-7698

Éditeur

APEF

Référence électronique

Éric Fougère, "Sa majesté des Mouches et les bienheureux de la désolation », Carnets [En ligne],

Deuxième série - 12 | 2018, mis en ligne le 19 janvier 2018, consulté le 19 avril 2019. URL : http://

journals.openedition.org/carnets/2465; DOI : 10.4000/carnets.2465

Ce document a été généré automatiquement le 19 avril 2019

\section{(c) (i) (8)}

Carnets est mis à disposition selon les termes de la licence Creative Commons - Atribution - Pas

d'utilisation commerciale 4.0 International. 


\section{Sa majesté des Mouches et les bienheureux de la désolation}

Deux cas de mimétisme insulaire aux antipodes?

\section{Éric Fougère}

1 En tout récit d'île, on peut mesurer des effets de résonance. Une hypothèse est de poser l'aptitude insulaire à reproduire à l'identique une société donnée. C'est d'autant plus vrai que les enfants du roman de William Golding et les ressortissants de Tristan da Cunha, dans le roman d'Hervé Bazin, sont des sujets britanniques, et que la Grande-Bretagne est elle-même une île. Une île en redouble une autre et la guerre que les enfants se livrent entre eux répète évidemment la conflagration qui préside à leur abandon: la bombe atomique éclate, ou c'est plutôt l'éruption d'un volcan qui préside à l'évacuation des habitants de Tristan vers la métropole anglaise, en sens inverse. Une hypothèse inverse est en effet de poser l'aptitude insulaire à non plus imiter mais différer. Sa Majesté des mouches est un roman de régression vers la barbarie par élection d'un bouc émissaire et par élimination de victimes allant jusqu'à frôler l'anéantissement collectif. À l'opposé, Les Bienheureux de La Désolation sont un roman de re-civilisation par injection d'une modernité que les déplacés vont adopter pour l'adapter, fondre en vue de la refonder.

2 Ma propre hypothèse, inspirée de René Girard, est que la volonté de différer revient en fait au besoin d'imiter. Le mimétisme instaure une identité différenciatrice où ce qui ressemble est en relation d'antagonisme et ce qui dissemble en relation de symétrie. Si bien que le roman de Bazin, loin de différer du modèle européen qu'il a la prétention de critiquer, pourrait bien le reconduire au contraire. De même que la régression des enfants n'est que la réduplication des impasses de la sauvagerie civilisée dont ils sont issus, quand l'île est incendiée par leur faute à la façon dont le monde en guerre est irradié par la bombe atomique, ainsi, les Tristans de Bazin sont loin d'imposer, malgré qu'ils en aient, la vision d'un progrès décanté grâce aux vertus d'une petite communauté syncrétique. En réalité, dans le procès mimétique entrepris par Bazin de manière enjouée (comme il l'est par Golding de manière apocalyptique), il se pourrait que l'emportent encore, ou du moins continuent de couver, les mécanismes d'exclusion que je me propose ici d'étudier. 


\section{Premiers fondements d'une exclusion}

3 Dans un premier temps le système est réinitialisé comme si de rien n'était. Pas de radeau pour enlever morceau par morceau les débris d'une épave à partir de quoi tout reprendre à zéro mais une conque appelant tous les enfants dispersés par le naufrage à se rassembler sur la plage. À quoi les voit-on s'occuper ? Comme avec le récit de la Genèse : à se nommer, se compter. Le dernier groupe à venir est celui d'un chœur de maîtrise en uniforme et tout ce qui vient revient donc à se constituer par imitation de la société des grandes personnes. Un second acte est l'élection d'un chef (A chief! A chief!). On procède au vote, en bonne démocratie. Mais ce premier mimétisme est déjà dissonant. Le chœur angélique évoque une armée d'ombres et l'analogie de cette armée suggère une horde de chasseurs. La petite société se reconstitue sous de mauvais auspices en relation de symétrie. Le garçon qu'on appelait Piggy (Cochonnet) dans la société de départ est immédiatement reconduit dans son ignominieux sobriquet par celui-là même auquel il a confié ce surnom sous le sceau du secret (Piggy! Piggy!). Simon, l'autre enfant défaillant, subit la même indiscrétion. Nous apprenons qu'il n'en est pas à son premier évanouissement. Dès leur apparition, les deux futures victimes du roman sont ainsi trahies respectivement par chacun des deux chefs «naturels », Ralph et Jack, et désignés tout de suite aux ricanements. Le mimétisme est d'ailleurs imparfait. L'asthme (asthma) de Piggy devient ass-mar (ass = cul + mar = gâté). Puis manque à l'appel un enfant, probablement tué. La ressemblance est minée par des conflits latents.

Dans le roman de Bazin, deux choses appellent une attention particulière. La première est le commandement d'allure juridico-biblique estampillant la démocratie tristan du sceau de la plus jalouse interdiction: «Nul ne s'élèvera ici au-dessus de quiconque » (Bazin, 1980 : 27). Un gouverneur est nommé, " pour la forme » (Ibid.), mais le règne est celui d'une "colonie chrétienne égalitaire " (Ibid.). On est apparemment très loin des conflits de rivalité puisqu'une égalité parfaite assure au " peuple élu des confins de la terre » (Bazin, 1980 : 183) une condition de semblables. Un fait est que l'indivision de la petite société (qui jamais «ne se divisa », Bazin, $1980: 28$ ) ne vient pas tant de son « entente » (Ibid.) et de son refus de la différenciation que d'une impossibilité d'y trouver qui pourrait différer : « les mous, les couards, les égoïstes et les inutiles sont inconnus » (Ibid.). Ce n'est pas que la persécution soit inconcevable, au contraire : il y a justement là tout un lot de victimes potentielles affublées de tous les attributs victimaires; c'est que la sélection naturelle a fait son œuvre. «La faim, l'inconfort, l'isolement, la lutte contre une nature hostile leur [aux Tristans] semblent aussi naturels que les saisons» (Ibid.). C'est que le dénuement des conditions de vie, par on ne sait quel paradoxe inexpliqué, fait que les Tristans sont dans un état de bonheur inaccessible aux accès du besoin: «le bonheur dans le dénuement, c'est une réussite ; c'est aussi une leçon vite tournée en scandale par ceux qu'humilie secrètement l'esclavage de leurs besoins » (Ibid.).

Nous sommes à peu près dans ce que le Rousseau du Discours sur l'origine et les fondements de l'inégalité parmi les hommes appelle « un juste milieu entre l'indolence de l'état primitif et la pétulante activité de notre amour-propre " et définit comme "l'époque la plus heureuse et la plus durable». Il attribue cette "véritable jeunesse du monde " à l'insularité :

De grandes inondations ou des tremblements de terre environnèrent d'eau ou de précipices des cantons habités; des révolutions du globe détachèrent et coupèrent 
en îles des portions du continent. On conçoit qu'entre des hommes ainsi rapprochés et forcés de vivre ensemble, il dut se former un idiome commun plutôt qu'entre ceux qui erraient librement dans les forêts de la terre ferme. (Rousseau, $1983: 140$ )

«Une île forcément, ça resserre tout » (Bazin, 1980 : 112). Ce que l'insularité fait ressortir est l'idée de communauté, bien résumée par un habitant parlant de Tristan : « ce n'est pas seulement une île, c'est nous » (Bazin, $1980: 59)$. Tout ce qui n'est pas «nous » porte un nom: les Extérieurs, aussi bien le dehors et ceux qui s'y trouvent. À l'interdiction de s'élever dans l'île au-dessus du niveau prescrit de stricte égalité s'ajoute en conséquence une idée d'exclusion constitutive. Et c'est le second point: quand les Tristans sont évacués, leur départ est marqué par une extermination des chiens de l'île afin d'y protéger le bétail et la basse-cour.

\section{Qui se ressemble s'assemble}

7 À Tristan, la similitude est une condition de l'égalité. La communauté n'est ce qu'elle est que dans la mesure où les insulaires y sont pareils. Égalité d'identité. Communauté de conformité. Le fils identifié ( $v$. Bazin, $1980: 84$ ) au père, et le commun reconnaissable à la continuité. C'est quand la comparaison survient que la raison du premier mimétisme (au sens de ressemblance) est à la lettre altérée: la compétition succède à la communion. Tristan da Cunha: qui se ressemble s'assemble. Angleterre: "qui se pose s'oppose " (Bazin, 1980 : 85). Une identité du même a fait son temps. Ce qu'il importe à présent de conquérir au contact de l'Autre est une identité de la différence. Or l'émulation d'une société de compétition ne produit, chez les insulaires exilés, que la simulation de gens déphasés dans un milieu qui n'est pas le leur. La solidarité, remplacée par un mouvement de charité, devient du « chacun chez soi » (Bazin, 1980 : 89). L'alternative est désormais la distinction (je me fais reconnaître) ou la dilution (je m'intègre). On lit ceci : «Notre groupe, après avoir été naturel, maintenu par des côtes, devenait artificiel, clos par un grillage, par la peur de se dissoudre » (Bazin, 1980 : 114). Un mimétisme est d'imitation (celui des Tristans dans leur île); un autre est de conflit, quand l'imitation cherche à se différencier par une appropriation triangulaire : A veut ce que $B$ possède en l'objet $C$, selon la définition bien connue de René Girard. Il s'agit de supprimer B, le médiateur et potentiellement rival, afin d'avoir un accès direct à l'objet du désir : «Il préférait se battre contre les choses que contre les gens et vivre au besoin plus durement, mais dans l'entraide » (Bazin, 1980 : 152), apprend-on d'un Tristan votant pour le retour à son île.

Chez Golding, le mimétisme est incarné par un couple de jumeaux nommé Samneric (troncation de Sam and Eric). Tout semble aller par deux dans le roman de Golding. La paire de lunettes de Piggy (qui finit cependant par ne plus voir que d'un seul œil en raison du bris d'un des verres) assure en apparence à la rivalité mimétique une structure exclusivement segmentaire ou duelle (et non triangulaire). Ainsi de la fonction détournée qui leur est dévolue pour allumer le feu. Car le feu présente à son tour une double fonction : faire évidemment de la fumée mais aussi donner le sentiment de sécurité d'un foyer. Le couteau possédé par Jack est bien sûr utile à la chasse. Il permet de manger de la viande à sa faim. Selon la perspective adoptée par René Girard dans Des choses cachées depuis la fondation du monde, on admettra que la chasse est une façon ritualisée de chercher des victimes à sacrifier. La chasse aux cochons devient de la sorte une chasse à l'homme où la fumée de l'incendie provoqué par les chasseurs à la poursuite de Ralph oblige celui-ci à détaler. La fumée salvatrice est alors homicide, et le double cri (double cry 
) des chasseurs est conçu pour à la fois se signaler réciproquement leur avance et refermer le cordon de feu sur la proie. Par-delà toutes les figures du double, il y a donc ambivalence et possibilité de retournement. « (...) what - Or else » (Golding, $1986: 188$ ) est une formule assez significative annonçant comment, du mimétisme éthologique, on passe à l'antagonisme anthropologique, et du segment (relation directe imitateur/imité) au triangle où l'objet du désir est la possession médiatisée de l'île (à la différence de ce qui se passe avec les Tristans, chez qui la volonté de possession de l'île est directe, immédiate).

Une façon d'aborder le meurtre fratricide perpétré par Caïn sur Abel est résumée par une phrase au détour d'un passage où Jack avoue ce qu'il ressent quand il chasse : «you can feel as if you're not hunting, but - being hunted » (Golding, 1986:57 - on a l'impression que le chasseur est le chassé). Comme si, précise-t-il, il y avait tout le temps quelque chose à tes trousses (" as if something's behind you all the time », Ibid.). Un peu plus loin dans le récit, nous voyons s'affronter pour la première fois Ralph et Jack, in love and hate (Golding, 1986 : 60). Un indéfinissable lien (indefinable connection) unit les deux rivaux mimétiques. Ils désirent en effet la même chose et ne la désirent, on le sait, que parce que cette chose est désirée par l'autre. On reconnaît là ce que René Girard écrit dans Mensonge romantique et vérité romanesque. Un personnage en est plus conscient que tous. Il s'agit de Piggy : « J'ai peur de lui, dit-il en substance en parlant de Jack, et c'est la raison qui fait que je le connais. La peur qu'on a de quelqu'un fait qu'on le déteste et qu'on pense à lui sans arrêt » (v. Golding, $1986: 102$ ). La question qui se pose, une fois posée l'identité des deux volontés rivales, est de se demander comment le mimétisme (au sens ici girardien) règle le sort du bouc émissaire en la personne de Piggy. "Je vais te dire une chose, ajoute ce dernier parlant à Ralph, il te déteste aussi. (...) c'est toi le chef et pas lui. (...) Je connais les gens. Je me connais. Je le connais. Il ne peut rien te faire à toi ; mais si tu ne tiens pas ton rang, c'est à moi qu'il s'en prendra » (Ibid.).

\section{Qui sacrifier ?}

10 Piggy réunit tous les attributs de la bête noire. Affecté de défauts de langage il est en même temps pourvu d'une intellectualité qui le distingue. Il a la vue courte en même temps qu'il est lucide. Il est à lui seul une objection vivante à la normalité. Surtout, pardelà son asthme et son embonpoint, ses lunettes et sa condition d'orphelin, Piggy porte un certain mimétisme à son comble. Il est naturellement le cochon que les enfants (qui se rendent invisibles en se badigeonnant la face aux couleurs de leur nouvel environnement) s'en vont tuer. C'est sur lui que va cristalliser la crise. Un transfert est opéré quand la conque, objet de médiation, devient l'enjeu du désir mimétique, et que Piggy, protecteur attitré de la conque, passe du statut de protégé de Ralph à celui de son unique allié. Piggy concentre à ce moment tous les traits de la persécution différenciatrice en nouant la rivalité mimétique à la peur collective. Toute l'histoire évolue du rose (« he went very pink ») au rouge ("he went red»). Le sang de Piggy se répand sur la roche ainsi que sur un autel. Il marche entre les javelots comme une victime au sacrifice en tenant la conque entre ses mains comme un objet rituel.

11 Exemplaire est la danse autour du feu dans le chapitre ayant pour titre $A$ view to a death (Aspect d'une mort). On apprête un festin. Ralph et Piggy paraissent, attirés par la présence humaine et par la faim. Les cuisiniers butent sur Piggy qu'ils brûlent avec leurs morceaux de viande encore chauds. « Ralph and the crowd of boys were united and relieved by a storm of laughter. Piggy once more was the centre of social derision so that everyone felt cheerful 
and normal » (Golding, 1986: 164 - un fou rire unit Ralph et la foule des garçons contre Piggy qui sert encore une fois d'exutoire, et la dérision permet à la communauté de se refaire un équilibre). Il y a des éclairs au milieu d'une obscurité terrifiante. Il fait un temps de tempête. Une mélopée s'organise en cercle au rythme d'un pouls général, au cri de «Tuer la bête !». Elle est de plus en plus frénétique. Un des enfants mime le cochon qu'on chasse avant de reculer laissant le centre du cercle vide: la place du sacrifice effectué sur Simon sortant de la forêt.

Piggy. Simon. Les deux victimes ont en commun leur assimilation sociale à « la chose » ou "la bête ». Ce qu'on ne peut nommer, du moins peut-on le localiser. Le «monstre " (indifférenciateur) est situé dans chacun des trois éléments que sont la terre, la mer et l'air. Il a trois réalisations successives : un soi-disant serpent qui s'enroule aux lianes de la jungle, un Léviathan supposé vivre au fond des mers, un parachute tombé du ciel où les combats aériens font rage à l'insu des enfants, qui voient dans la dépouille du parachutiste accroché dans les branches une sorte de gorille. À chacune de ces peurs on réagit par une violence sacrificielle entretenant quelque homologie de forme avec l'objet de la peur. Ainsi de la tête de porc fixée sur une pique en guise d'offrande évoquant la position de pantin désarticulé du parachutiste avec sa tête entre les genoux. Qu'est-ce que mangerait la bête ? interroge en toute incrédulité Piggy. Du cochon, lui répond-on. Nous mangeons du cochon. Deux logiques ont beau s'affronter (les causes naturelles et les effets psychologiques), on sait que l'opposition n'est qu'apparente. La Chose est intérieure, et la bête est l'homme.

13 À Simon, l'oracle affreux dit, par la bouche ensanglantée du cochon propitiatoire et totémique : "There isn't anyone to help you. Only me. And I'm the Beast. (...) I'm part of you. (...) I'm the reason of why it's no go. Why things are what they are. " (Golding, 1986: 158 - il n'y a personne ici pour t'aider. Que moi. Qui suis la Bête. Je fais partie de toi. Je suis ce qui fait que rien ne va. Ce qui fait que les choses sont ce qu'elles sont). Simon n'est pas tué, comme Piggy, de manière intentionnelle. Il l'est par surprise et par méprise : on le prend pour la bête sortant des bois. C'est qu'il n'est impliqué directement ni dans la persécution ni dans la médiation mimétique : il aime tout le monde et la solitude est son lot. Mais il est précisément le seul à qui le secret de la crise indifférenciatrice est révélé. Le seul à faire le lien des causes réelles et des développements monstrueux (monstre = bête + peur). Le seul à voir la fausse et la vraie Bête en face. Il voit la vraie (tête du cochon décapité), mais il démystifie la fausse : il sait que le parachutiste est une pauvre chose abîmée (poor broken thing). Les deux reviennent au même au prix d'une inversion : si le parachutiste était un homme, alors l'homme est ce cadavre. On meurt de ce genre de vérité. Le Deus ex machina sauve en partie d'une annulation: l'incendie ravageant l'île y conduit un officier de la marine anglaise. En partie, car il s'agit de la marine de guerre, et l'île est en flammes. " Awful things has been done on this island " (Golding, 1986:188 - il s'est passé des choses horribles sur cette île), avait prévenu Piggy. Le premier matin remonte à la nuit des temps (« that first morning ages ago », Ibid.).

Les chiens massacrés font les frais de l'évacuation de Tristan; les vieux de l'île, évincés, font ceux du rapatriement. L'expérience anglaise a révélé la supériorité technologique occidentale. On va l'importer dans Tristan «sans en devenir esclaves»: «On aimerait aussi, nous les jeunes, intervenir plus souvent. Nous le pouvons maintenant. Ce que nous avons appris, au stage, ne nous qualifie guère en Angleterre, où il y a mieux. Mais à Tristan, notre chance devient meilleure parce que, déjà, nous surclassons les vieux " (Bazin, 1980: 152), dit l'un des jeunes. À l'égalité d'identité fondée sur un rang 
d'ancienneté succède une égalité de rapport et de représentation tirée d'un choix de compétence. Il y a donc un bouleversement. Si ce bouleversement n'est pas critique (aux deux sens du mot), c'est qu'il est réalisé sans violence. À la compétition, qui met des antagonistes à la fois distincts et réunis dans la même identité de comportement pour avoir le dessus, répond la version édulcorée du jeu. C'est ainsi que les matchs organisés désormais sur Tristan se jouent sans adversaire : on ne joue pas contre, on joue, dit le texte, «ensemble " (Bazin, 1980: 199). Où l'excès de sérieux par imitation des vieux rendait le jeu pur impossible ${ }^{1}$, avant la parenthèse anglaise, il y a maintenant des équipes adonnées au foot : « elles se sont constituées sur le terrain même, de ce que leur offrait la jeunesse - adultes ou gamins de toutes tailles répartis en fonction des performances et des nécessités » (Bazin, 1980 : 198-199). Moyennant quoi la donne a bien changé. Puisés dans le "fonds commun» (Bazin, 1980: 199), les joueurs appartiennent, encore et toujours, à la " communauté d'abord» (Ibid.); il y a, cependant, que l'uniformité passée fait maintenant place à la diversité des âges et des maillots. La compétence acquise ailleurs appelle aussi, sur le terrain, les « performances ».

Au-delà la réussite apparente, on ne peut s'empêcher d'être inquiet pour l'équilibre atteint. Rien de moins, rien de plus : « il ne faut que ce qu'il faut » (Bazin, $1980: 201$ ), dit un bienheureux de la " confrérie satisfaite » (Bazin, $1980: 233)$. Ou " trop est trop » (Ibid.), dit le même à l'intention d'un étranger venu visiter l'île dans la tradition d'utopie la plus orthodoxe. Que dit la tautologie? D'abord une circularité bien close, à l'image d'une île autarcique, et qui ne l'est plus. Telle est la brèche ouverte au sein de la perfection :

Si l'Angleterre n'avait pas fait du sentiment, si la langouste ne se vendait pas en bonnes devises, si vous n'étiez pas bien placé au milieu de la grande baille pour offrir votre météo ou votre radio, si la vente des timbres et le tampon de complaisance pour philatélistes ne fournissaient pas à Sainte Albion de quoi entretenir son staff, croyez-vous que vous en seriez là ? (Bazin, 1980 : 232),

demande un observateur.

Ensuite une identité qui ne souffre aucune contradiction : la tautologie dit une chose et la même et ne dit rien d'autre autrement qu'en les répétant terme à terme. On répliquera que la restriction négative (ou la négation partielle) « il ne faut que ce qu'il faut » n'est pas rigoureusement tautologique à cet égard et que trompeuse est sa fausse identité, mais c'est bien la même idée de clôture insulaire et de circularité de l'énoncé qu'il y a derrière : un « isolement (...) nous protège du luxe en le rendant hors de prix » (Bazin, $1980: 233$ ). Nous savons que l'isolement n'est pas complet, d'une part, et nous n'ignorons pas, d'un autre côté, que le luxe est hors de prix par définition. Tout se passe en effet non seulement comme si l'entente intestine interdisait toute opposition ${ }^{2}$ mais comme si, derrière un gentil petit roman de Bienheureux, couvait la Désolation d'un texte effacé sous le palimpseste utopique. On en voudrait pour indication le nom de Désolation, qui s'applique en général aux lugubres îles Kerguelen, terres de naufrage. On est ainsi rabattu, par-delà les propos lénifiants, sur le scénario girardien de Sa Majesté des mouches.

\section{Girard or not Girard?}

Dans La Postérité de Robinson, Jean-Paul Engélibert essaie de tirer le roman de Golding vers une interprétation qui relierait Sa Majesté des mouches à Totem et tabou. Cette optique amène à discréditer l'entreprise civilisatrice (asthmatique autant qu'anale et refusant l'Éros) et à valoriser la tribu de Jack : « un socius fondé sur la reconnaissance du désir, de 
l'autorité d'un chef, et qui culminera dans la formation d'un culte et l'élaboration d'une esthétique rudimentaire. (...) une société dotée de règles et de rites » (Engélibert, 1997 : 185). En régressant vers la barbarie, le roman progresserait vers l'élucidation de «l'essence anhistorique du lien social» et, ce faisant, vers «le crime fondateur de la civilisation» (Engélibert, 1997: 186). Nous sommes ainsi, malgré l'enracinement du roman dans le réel historique avec la Seconde Guerre mondiale au point de départ et d'arrivée, ramenés vers Totem et tabou de Freud en vertu de la " puissance sexuelle » (Ibid.) qui se dégage d'abord de Ralph et, dans un second temps, de la prise en compte, incarnée par Jack, de la réalité libidinale et du «meurtre originel qui soude la communauté en la dotant d'un père symbolique » (Engélibert, 1997 : 187).

L'intérêt d'une telle relecture est d'accréditer l'idée de fiction des origines. Une note expose le parti pris de Jean-Paul Engélibert : «Cette interprétation permet de réévaluer les thèses de Totem et tabou sans les dissocier du socle de la théorie freudienne, alors que Girard, sans qu'on comprenne très bien pourquoi, soustrait ce livre à la psychanalyse dont il refuse les autres apports » (Engélibert, 1997 : 177). On sait pourtant bien ce que René Girard incrimine. Alors qu'œedipe selon Freud est, dans Totem et tabou, le " complexé » psychanalytique, il en va tout autrement chez René Girard. Autant l'un fait s'apparenter le totem au père et justifie le tabou de l'inceste en faisant coïncider les deux crimes œdipiens dans ce qui serait une psyché collective, autant l'autre insiste au contraire sur la réalité victimaire en faisant d'ÆEdipe un bouc émissaire historique identifiable en contexte de peste. Le parricide et l'inceste y sont bien des conditions nécessaires; elles ne sont pas suffisantes. "Les persécuteurs croient choisir leur victime en vertu des crimes qu'ils lui attribuent et qui font d'elle, à leurs yeux, la responsable des désastres auxquels ils réagissent par la persécution. En réalité ce sont des critères persécuteurs qui les déterminent» (Girard, 2005 : 42), écrit René Girard dans Le Bouc émissaire évoquant la boiterie d'œEdipe et son passé d'enfant exposé, sa situation d'étranger, sa qualité de roi parvenu, comme autant de ces critères.

On doit, de même, insister sur l'historicité des Bienheureux de La Désolation. René Bazin fait tout pour en hypothéquer la charge au profit de ce qui pourrait passer pour un «conte philosophique »(Bazin, 1980 : 241). Il préfère à la bombe atomique un volcan, substituant une catastrophe naturelle à la guerre, alors même que des essais nucléaires ont eu lieu secrètement dans les eaux de Tristan da Cunha pendant la Guerre froide. Il a beau mythifier, comme Engélibert après Freud, une opération de fin d'un monde aux fins de refondation de ce monde, on se souvient que les deux camps de réfugiés du roman, conformes à l'Histoire en cela, sont d'anciennes bases militaires elles-mêmes désaffectées (Pendell Army Camp et Calshot). Il faut donc en revenir à René Girard, à l'insistance avec laquelle il soutient, contre Freud ou Lévi-Strauss, que les mythes appartiennent à l'histoire persécutrice. En posant l'égalité de principe et de fait à Tristan, le roman de Bazin coupe apparemment l'herbe sous le pied de la rivalité, dans un contexte historique et d'écriture (années 60-70) où le mot d'ordre est de condamner le pouvoir et la société de consommation. Mais, si «toutes les sociétés (...) sont des îles », insinue le porte-parole du roman, «nulle ne convainc l'autre de vivre comme elle» (Bazin, 1980 : 240). Il y aurait donc un conflit sous-jacent. C'est que le conflit girardien n'est pas d'inspiration sociologique, il est d'essence anthropologique. Une société d'égaux peut être une société de rivaux. Doit nécessairement l'être, en démocratie, nous dit Tocqueville avant Girard. À moins d'envisager l'homme en régime totalitaire. Alors, on serait moins dans le cas d'une 
égalité que dans celui d'une identité. Les Bienheureux de la Désolation flottent en permanence entre ces deux pôles, ou sociologique ou anthropologique.

\section{En conclusion}

L'identité renforce évidemment le mimétisme : identité de lieu (l'île), identité de situation (la catastrophe), identité d'action (la survie). La transplantation commune aux récits de Golding et de Bazin cause un choc. Il en naît le besoin de reproduire à l'identique une organisation secouée par les nécessités de la confrontation. Sous la pression, la tribu des enfants respecte un scénario typiquement girardien qui va de la crise à l'élimination d'un bouc émissaire. L'objet du désir étant le pouvoir, il divise. Il ne le fait pas chez les Tristans, dont, par un redoublement, le moteur est leur identité même. Ils ne sont pas seulement tout entiers définis comme identiques entre eux (moins des prochains que des semblables) ; ils veulent aussi devenir identiques à eux-mêmes au sein du changement qui les fait rester ce qu'ils sont, pareils. Où la ressemblance est à ce point une valeur, le mimétisme est sans matière. Il est sans prise. Il tourne à vide. Il est tautologique. On voit certes une volonté de différer du modèle anglais, mais ce modèle est approprié sur un mode où la technique est, soi-disant, seule intégrée. Ce modèle est en réalité désintégré, comme il en va du roman de Bazin, privé de dynamique interne et prisonnier de son oxymorique impossibilité : des bienheureux de la désolation.

\section{BIBLIOGRAPHIE}

BAZIN, Hervé (1980). Les Bienheureux de la Désolation. Paris : Seuil (Points roman).

ENGÉLIBERT, Jean-Paul (1997). La Postérité de Robinson : un mythe littéraire de la modernité (1954-1986). Genève : Droz.

FREUD, Sigmund (1977). Totem et tabou. Paris : Petite Bibliothèque Payot.

GIRARD, René (1961). Mensonge romantique et vérité romanesque. Paris : Grasset.

GIRARD, René (1978). Des choses cachées depuis la fondation du monde. Paris : Grasset.

GIRARD, René (2005). Le Bouc émissaire. Paris : Grasset, Librairie générale française (Biblio essais).

GOLDING, William (1986). Lord of the Flies. London-Boston: Faber and Faber.

ROUSSEAU, Jean-Jacques (1983). Discours sur l'origine et les fondements de l'inégalité parmi les hommes. Paris : Éditions sociales.

\section{NOTES}

1. «Impossible de leur faire admettre une convention de jeu, murmure Miss Gow. Je n'ai jamais vu d'enfants si cramponnés au réel. (...) Pour les plus grands, dont s'occupe un chef scout des 
environs, c'est à peine différent. Ils font du feu, montent une tente, enlèvent un canot d'un coup de rame, en petits adultes qui chez eux le font 'pour de vrai'« (Bazin, $1980: 84$ ).

2. «Ce qui nous classe à part, je crois, c'est que depuis cinquante ans, du fait de la nécessité, l'opposition nous est devenue moins naturelle que l'entente » (Bazin, 1980 : 225).

\section{RÉSUMÉS}

Dans Sa Majesté des mouches et dans Les Bienheureux de la Désolation, Golding et Bazin semblent aux antipodes. Ici, l'éruption d'un volcan fait évacuer les habitants de Tristan da Cunha vers l'Angleterre, et là, c'est la déflagration d'une bombe et la conflagration d'une guerre mondiale qui font que des enfants britanniques se trouvent abandonnés sur une île. Autant ces derniers régressent à l'état sauvage en se choisissant des chefs en relation de rivalité mimétique, autant les premiers respectent une égalité qui les prémunit normalement contre tout antagonisme. On voit cependant que le besoin d'imiter le modèle anglais, chez les enfants naufragés, les amène à s'en différencier par éclatement du groupe et par élimination d'un bouc émissaire, et que la volonté de différer de ce modèle, avec les habitants de Tristan finalement rapatriés, les conduit eux-mêmes à exclure ce qui dérange une identité farouchement différenciatrice en apparence, en réalité parfaitement mimétique.

At first sight, Golding's Lord of the Flies and Bazin's Les Bienheureux de la Désolation would seem to be at opposite poles. In Bazin's work the inhabitants of Tristan da Cunha are evacuated to England to escape a volcanic eruption; Golding, by contrast, has a group of British schoolchildren stranded on an uninhabited island after being caught up in a global conflict. And whereas the latter revert to a primitive state by choosing leaders who adopt postures of mimetic rivalry, the Tristan islanders remain true to an egalitarian tradition that frowns on any open display of antagonism. In the event, however, it is precisely the children's emulation of their English model that results in their breaking away from it by forming competing groups and eliminating one of their number as a scapegoat, while it is the evacuees' commitment to a different model that leads them to reject anything that compromises an identity which, though highly distinctive in appearance, is in point of fact wholly mimetic.

31 janvier 2018

\section{INDEX}

Mots-clés : civilisation, sauvagerie, mimétisme, bouc émissaire, île

Keywords : civilization, primitive state, mimetism, scapegoat, island

\section{AUTEUR}

\section{ÉRIC FOUGÈRE}

Lycée Saint-Exupéry (Marseille)

eric.fougere98[at]gmail.com 